\title{
PATTERNS OF ANTIMICROBIAL RESISTANCE AMONG INTENSIVE CARE UNIT PATIENTS OF A PRIVATE MEDICAL COLLEGE HOSPITAL IN DHAKA
}

\author{
QUAZI TARIKUL ISLAM ${ }^{1}$, MD. MAHMUDUR RAHMAN SIDDIQUI ${ }^{2}$, FARHANA RAZ $^{3}$, \\ MOHAMMAD ASRAFUZZAMAN ${ }^{4}$, MD. ROBED AMIN ${ }^{5}$
}

\begin{abstract}
:
Because of importance of Hospital acquired infections (HAIs), it is critical to conduct surveillance studies to obtain the required data about the regional microorganisms and their susceptibility to antibiotics. This study to investigate antimicrobial resistance pattern among Intensive Care Unit (ICU) patients in a private medical college hospital setup. In a cross sectional study, 100 specimens from patients admitted in the ICU who had signs or symptoms of nosocomial infection were collected from 2012 - 2013. For each patient, samples of blood, urine, tracheal aspirate, sputum, wound swab, pus, and endotracheal tubes were obtained, cultured and analyzed with antibiogram. The most common primary diagnosis were aspiration pneumonia (49\%) and UTI (20\%) respectively. The most common locations for infection were tracheal aspirate (54\%). The most frequent gram negative microorganisms derived from samples were Acinetobacter spp (29\%), Klebsiella spp (26\%) and Pseudomonas spp (18\%). Klebsiella spp, Acinetobacter spp and Pseudomonas spp were most common resistant organisms among all. Klebsiella spp were resistant against Ceftriaxone (84.6\%), Ceftazidime (82.6\%), Amikacin (46.1\%), Gentamicin (66.6\%) and Quinolones (65-66.6\%) respectively. Acinetobacter spp were resistant against Ceftriaxone (85\%), Ceftazidime (88.8\%), Cefotaxime (85.7\%), Meropenem (79.3\%),Amikacin (86.2\%), Gentamicin (84.5\%) and Quinolons (86.2-89.2\%) respectively. Pseudomonas spp were resistant against Ceftriaxone (70.5\%), Ceftazidime (66.6\%), Amikacin (68.7\%), Gentamicin (58.8\%), Meropenem (52.9\%) and Quinolones (81.2-86.6\%) respectively. Meropenem was the most sensitive antibiotic against Klebsiella spp (84.6\%) but Cotrimoxazole in case of Acinetobacter spp (60\%) respectively. Escherichia coli were mostly isolated from urine, which was sensitive to Amikacin (73.3\%) and Meropenem (86.6\%) respectively. Gram-negative pathogens obtained from ICU patients in our settings show high resistance to antibiotics. Regular monitoring of the pattern of resistance of common pathogens in the ICUs is essential to up-to-date the use of rational antibiotics regiments.
\end{abstract}

Key words: Antibiotic, microorganism, nosocomial infection, Healthcare Associated Infections, antimicrobial resistance.

Received: 20 January 2014

Accepted: 19 June 2014

\section{Introduction :}

Infectious diseases are major health problems in Bangladesh requiring frequent use of antimicrobials. ${ }^{1}$ Antibiotic-resistant bacteria are a big threat among critically ill patients. Infections in critically ill patients exert a high rate of morbidity and mortality. Because of significant underlying disease processes and deranged physiologic status, critically ill patients are more susceptible to various infections and adverse outcome from infection. ${ }^{2}$ Many infectious diseases do not respond to conventional antimicrobial regiments. Standard treatment guidelines of different microbes are not sufficient for the purpose. ${ }^{3}$
Healthcare Associated Infections (HAIs) are especially important in intensive care units (ICUs) where they have a five-fold higher incidence rate compared to the general inpatient population. ${ }^{4}$ This is due to the increased use of invasive medical instruments such as mechanical ventilators, monitoring devices, blood, urine catheters, which in turn is a result of overt use of broad-spectrum antibiotics. ${ }^{5}$ These antimicrobial resistant patterns are always changing with time. Because of importance of HAIs, it is critical to conduct surveillance studies to obtain the required data about the regional microorganisms and their susceptibility to antibiotics. ${ }^{3}$ This study is aimed to provide such information for our clinicians.

1. Professor of Medicine, Popular Medical College \& Hospital, Dhaka.

2. Assistant Professor of Medicine, Anwer Khan Modern Medical College \& Hospital, Dhaka.

3. RMO, Intensive Care Unit (ICU), Popular Medical College \& Hospital, Dhaka.

4. In charge, Intensive Care Unit (ICU), Popular Medical College \& Hospital, Dhaka.

5. Assoc Prof of Medicine,Dhaka Medical College \& Hospital, Dhaka. 


\section{Material \& Methods:}

In this cross-sectional study, from 2012 to 2013, we collected 100 specimens from patients with criteria of HAIs infection, admitted in the intensive care unit of a Private Medical College Hospital in Dhaka. For each patient, an structured case record form was filled. Clinical specimens included blood, urine, pus and discharges from endotracheal tubes and post surgical wounds swabs, were collected and cultured on Eosin Methylen Blue (EMB), Blood agar, chocolate agar, thioglycollate and Trypticase Soy broth (TSB) media and incubated at $37^{\circ} \mathrm{C}$ for $24-48 \mathrm{~h}$. Thioglycollate cultures and TSB bottles were re incubated for at least 7 days and subcultured on EMB and blood agar or chocolate agar plates, as necessary. For each patient, samples of blood, urine, tracheal aspirate, sputum, wound swab, pus, and endotracheal tubes were obtained, cultured and analyzed with antibiogram. The study protocol was approved by research ethics committee of Institute and each patient's family gave written informed consent before enrollment the study. The data was analyzed using Statistical Package for the Social Sciences (SPSS) version 16 Chicago illionois.

\section{Results:}

From the total 100 specimens obtained, 67\% were from male patients and 33\% from females. $30 \%$ of the patients were within the 61-70 years of age group and $18 \%$ were within $>80$ years age group respectively [Figure 1]. The most common primary diagnosises were aspiration pneumonia (49\%) and UTI (20\%) respectively [Table-2]. The most common locations for infection were tracheal aspirate (54\%), urine (20\%), blood $(10 \%)$ and sputum (8\%) [Table-1]. The most frequent microorganisms derived from samples were Acinetobacter spp (29\%), Klebsiella spp (26\%) and Pseudomonas spp (18\%), Escherichia coli (15\%), Staphylococcus aureus (6), Streptococcus (4\%), Salmonella spp (1\%) and Morganella morganii (1\%) respectively [Table-II]. Acinetobacter spp, Klebsiella spp and Pseudomonus spp were mostly found in sample from tracheal aspirate. But Escherichia coli was mostly isolated from urine samples [Figure-2].
Table III, IV, V explains the sensitivity of different microorganisms to common antibiotics. Klebsiella spp, Acinetobacter spp and Pseudomonas spp were most common resistant organisms among all. In table 3 out of 95 samples $77 \%$ samples were resistant to ceftriaxone and $73 \%$ were to Ceftazidime respectively. These organisms were mostly Pseudomonas spp, Klebsiella spp, Acinetobacter spp respectively. Klebsiella spp were resistant against Ceftriaxone (84.6\%), Ceftazidime (82.6\%), Amikacin (46.1\%), Gentamicin (66.6\%) and Quinolones (65-66.6\%) respectively [Table-III, IV, V]. Acinetobacter spp were resistant against Ceftriaxone (85\%), Ceftazidime (88.8\%), Cefotaxime (85.7\%), Meropenem (79.3\%), Amikacin (86.2\%), Gentamicin (84.5\%) and Quinolons (86.2-89.2\%) respectively [TableIII, IV, V]. Pseudomonas spp were resistant against Ceftriaxone (70.5\%), Ceftazidime (66.6\%), Amikacin (68.7\%), Gentamicin (58.8\%), Meropenem (52.9\%) and Quinolones (81.2-86.6\%) respectively [Table-III, IV, V]. Meropenem was the most sensitive antibiotic against Klebsiella spp (84.6\%) but Acinetobacter spp were still resistant to Meropenem and Amikacin but sensitive to Cotrimoxazole (60\%) [Table- V]. Table 4, 5 showed Escherichia coliwere mostly sensitive to Amikacin (73.3\%) and Meropenem (86.6\%) respectively. Gram-negative pathogens obtained from ICU patients in our settings show high resistance to antibiotics.

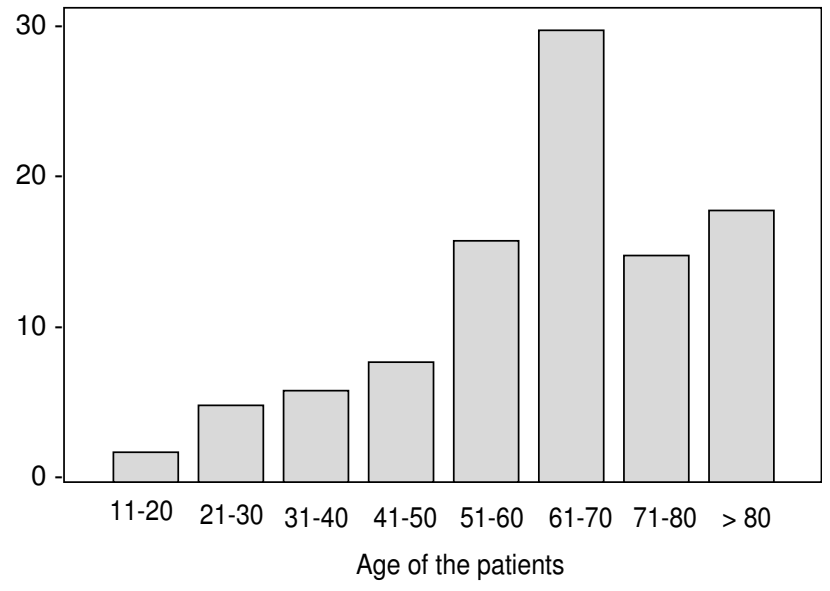

Fig.-1: Age distribution of the patients.

Table-I

Frequency of different microorganisms from various samples $(n=100)$.

\begin{tabular}{lcccccccc}
\hline Microorganism & Blood & Urine & $\begin{array}{c}\text { Tracheal } \\
\text { Aspirate }\end{array}$ & Sputum & $\begin{array}{c}\text { Wound } \\
\text { Swab }\end{array}$ & $\begin{array}{c}\text { Pus } \\
\text { Endotrachial }\end{array}$ & $\begin{array}{c}\text { Tube } \\
\text { Total (\%) }\end{array}$ \\
\hline Acinetobacter spp & $3(10.3)$ & $1(3.4)$ & $20(68.9)$ & $2(6.8)$ & $2(6.8)$ & 0 & $1(3.4)$ & $29(29)$ \\
Klebsiella spp & $3(11.5)$ & $3(11.5)$ & $16(61.5)$ & $2(7.6)$ & 0 & $2(7.6)$ & 0 & $26(26)$ \\
Pseudomonas spp & $1(5.5)$ & $4(22.2)$ & $9(50)$ & $3(16.6)$ & 0 & 0 & $1(5.5)$ & $18(18)$ \\
Escherichia coli & $2(13.3)$ & $7(46.6)$ & $4(26.6)$ & 0 & $1(6.6)$ & $1(6.6)$ & 0 & $15(15)$ \\
Staphylococcus aureus & 0 & 0 & $5(83.3)$ & $1(16)$ & 0 & 0 & 0 & $6(6)$ \\
Streptococcus & 0 & $4(100)$ & 0 & 0 & 0 & 0 & 0 & $4(4)$ \\
Salmonella spp & $1(100)$ & 0 & 0 & 0 & 0 & 0 & 0 & $1(1)$ \\
Morganella morganii & 0 & $1(100)$ & 0 & 0 & 0 & 0 & $1(1)$ \\
\hline Total & $\mathbf{1 0 ( 1 0 )}$ & $\mathbf{2 0 ( 2 0 )}$ & $\mathbf{5 4 ( 5 4 )}$ & $\mathbf{8 ( 8 )}$ & $\mathbf{3 ( 3 )}$ & $\mathbf{3 ( 3 )}$ & $\mathbf{2 ( 2 )}$ & $\mathbf{1 0 0 ( 1 0 0 )}$ \\
\hline
\end{tabular}




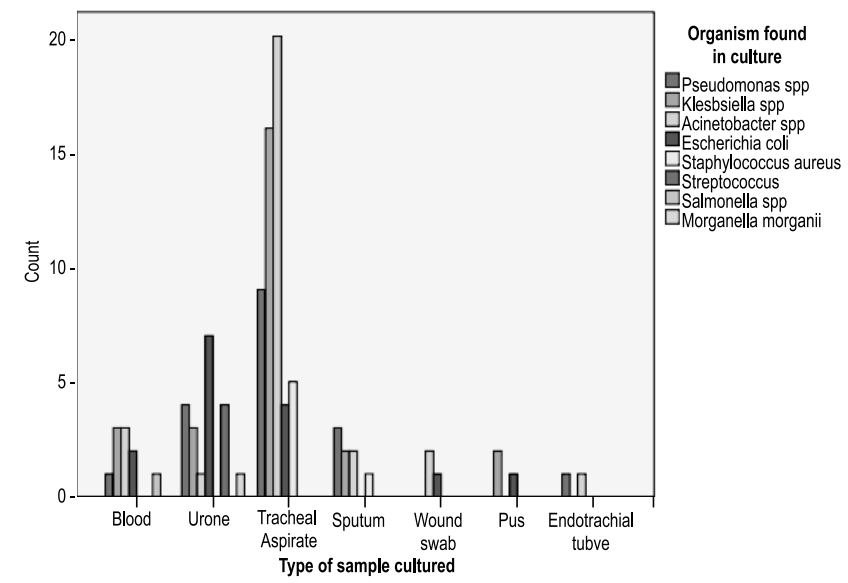

Fig.-2 :Distribution of various microorganisms according to types of samples cultured.
Table-II

Primary diagnosis of these ICU patients. $(n=100)$

\begin{tabular}{lcc}
\hline Primary Diagnosis & Frequency & Percentage \\
\hline Aspiration Pneumonia & 49 & 49.0 \\
UTI & 20 & 20.0 \\
Septicaemia & 11 & 11.0 \\
Pneumonia & 10 & 10.0 \\
COPD with respiratory failure & 4 & 4.0 \\
Surgical wond infection & 3 & 3.0 \\
Empyma thoracic & 3 & 3.0 \\
\hline Total & 100 & 100.0 \\
\hline
\end{tabular}

Table-III

Distribution of microorganisms according to susceptibility to penicillin and cephalosporins groups of antibiotics. $(n=100)$

\begin{tabular}{|c|c|c|c|c|c|c|c|c|c|c|}
\hline \multirow[t]{2}{*}{ Microorganism } & \multicolumn{2}{|c|}{$\begin{array}{c}\text { Amoxicillin } \\
(\%)\end{array}$} & \multicolumn{2}{|c|}{$\begin{array}{c}\text { Piperacillin+ } \\
\text { Tazobactum (\%) }\end{array}$} & \multicolumn{2}{|c|}{$\begin{array}{c}\text { Ceftriaxone } \\
(\%) \\
\end{array}$} & \multicolumn{2}{|c|}{$\begin{array}{c}\text { Ceftazidime } \\
(\%)\end{array}$} & \multicolumn{2}{|c|}{ Cefotaxime } \\
\hline & $\mathrm{S}$ & $\mathrm{R}$ & $\mathrm{S}$ & $\mathrm{R}$ & $\mathrm{S}$ & $\mathrm{R}$ & $\mathrm{S}$ & $\mathrm{R}$ & $\mathrm{S}$ & $\mathrm{R}$ \\
\hline Acinetobacter $s p p$ & $\mathrm{O}(0)$ & $1(100)$ & $2(66.6)$ & $1(33.3)$ & $4(14.8)$ & $23(85.1)$ & $3(11.1)$ & $24(88.8)$ & $2(14.2)$ & $12(85.7)$ \\
\hline Klebsiella spp & $\mathrm{O}(0)$ & $2(100)$ & $1(33.3)$ & 2 (66.6) & $4(15.3)$ & $22(84.6)$ & $4(17.3)$ & 19 (82.6) & $2(28.5)$ & $5(71.4)$ \\
\hline Pseudomonas spp & $\mathrm{O}(0)$ & $3(100)$ & $1(50)$ & $1(50)$ & $5(29.4)$ & $12(70.5)$ & $6(33.3)$ & $12(66.6)$ & $5(45.4)$ & $6(54.5)$ \\
\hline Escherichia coli & - & - & $2(66.6)$ & $1(33.3)$ & $3(20)$ & $12(80)$ & $5(35.7)$ & $9(64.2)$ & $1(50)$ & $1(50)$ \\
\hline Staphylococcus aureus & - & - & $2(100)$ & $0(0)$ & $0(0)$ & $4(100)$ & $0(0)$ & $5(100)$ & - & - \\
\hline Streptococcus & $1(50)$ & $1(50)$ & - & - & $1(25)$ & $3(75)$ & $1(25)$ & $3(75)$ & - & - \\
\hline Salmonella spp & - & - & - & - & $1(100)$ & $0(0)$ & $1(100)$ & $0(0)$ & - & - \\
\hline Morganella morganii & $\mathrm{O}(0)$ & $1(100)$ & - & - & $0(0)$ & $1(100)$ & $0(0)$ & $1(100)$ & $0(0)$ & $1(100)$ \\
\hline Total & $1(11.1)$ & $8(88.8)$ & $8(61.5)$ & $5(38.4)$ & $18(21.1)$ & $77(81)$ & $20(21.5)$ & $73(78.4)$ & $10(28.5)$ & $25(71.4)$ \\
\hline
\end{tabular}

Note: S: antibiotic sensitive, R: antibiotic resistant.

Table-IV

Distribution of microorganisms according to susceptibility to aminoglycosides, macrolides and lincosamides groups of antibiotics. $(n=100)$

\begin{tabular}{|c|c|c|c|c|c|c|c|c|c|c|}
\hline \multirow{2}{*}{ Microorganism } & \multicolumn{2}{|c|}{ Amikacin } & \multicolumn{2}{|c|}{ Gentamicin } & \multicolumn{2}{|c|}{ Netilmicin } & \multicolumn{2}{|c|}{ Azithromycin } & \multicolumn{2}{|c|}{ Clindamycin } \\
\hline & $\mathrm{S}$ & $\mathrm{R}$ & $\mathrm{S}$ & $\mathrm{R}$ & $\mathrm{S}$ & $\mathrm{R}$ & $\mathrm{S}$ & $\mathrm{R}$ & $\mathrm{S}$ & $\mathrm{R}$ \\
\hline Acinetobacter spp & $4(13.7)$ & $25(86.2)$ & $4(16.6)$ & $20(84.4)$ & $5(19.2)$ & $21(80.7)$ & 0 & $1(100)$ & $1(50)$ & $1(50)$ \\
\hline Klebsiella spp & $14(53.8)$ & $12(46.1)$ & $8(33.3)$ & 16(66.6) & $8(40)$ & $12(60)$ & 0 & $2(100)$ & 0 & $2(100)$ \\
\hline Pseudomonas spp & $5(31.2)$ & $11(68.7)$ & $7(41.1)$ & $10(58.8)$ & $8(50)$ & $8(50)$ & $1(25)$ & $3(75)$ & 1(16.6) & $5(84.4)$ \\
\hline Escherichia coli & $11(73.3)$ & 4(26.6) & $7(50)$ & $7(50)$ & $8(57.1)$ & $6(42.8)$ & - & - & - & - \\
\hline Staphylococcus aureus & s $1(20)$ & $4(80)$ & $1(16.6)$ & $5(84.4)$ & 0 & $4(100)$ & - & - & - & - \\
\hline Streptococcus & 0 & $3(100)$ & $1(25)$ & $3(75)$ & 0 & $3(100)$ & 0 & $1(100)$ & 0 & $1(100)$ \\
\hline Salmonella spp & - & - & $1(100)$ & 0 & - & - & - & - & - & - \\
\hline Morganella morganii & 0 & $1(100)$ & 0 & $1(100)$ & 0 & $1(100)$ & 0 & $1(100)$ & 0 & $1(100)$ \\
\hline Total & $35(36.8)$ & $60(63.1)$ & $29(31.1)$ & $64(68.8)$ & $29(34.5)$ & $55(65.4)$ & $1(11.1)$ & $8(88.8)$ & $2(16.6)$ & $10(84.4)$ \\
\hline
\end{tabular}

Note: S: antibiotic sensitive, R: antibiotic resistant. 
Table-V

Distribution of microorganisms according to susceptibility to quinolon and other groups of antibiotics. ( $n=100)$

\begin{tabular}{|c|c|c|c|c|c|c|c|c|c|c|}
\hline \multirow[t]{2}{*}{ Microorganism } & \multicolumn{2}{|c|}{ Ciprofloxacin(\%) } & \multicolumn{2}{|c|}{ Levofloxacin(\%) } & \multicolumn{2}{|c|}{ Meropenem(\%) } & \multicolumn{2}{|c|}{ Colistin(\%) } & \multicolumn{2}{|c|}{ Cotrimoxazole(\%) } \\
\hline & $\mathrm{S}$ & $\mathrm{R}$ & $\mathrm{S}$ & $\mathrm{R}$ & $\mathrm{S}$ & $\mathrm{R}$ & $\mathrm{S}$ & $\mathrm{R}$ & $\mathrm{S}$ & $\mathrm{R}$ \\
\hline Acinetobacterspp & $3(10.7)$ & $25(89.2)$ & $4(13.7)$ & $25(86.2)$ & $6(20.6)$ & $23(79.3)$ & $2(40)$ & $3(60)$ & $12(60)$ & $8(40)$ \\
\hline Klebsiella spp & $8(33.3)$ & $16(66.6)$ & $8(34.7)$ & $15(65.2)$ & $22(84.6)$ & $4(15.3)$ & $2(66.6)$ & 1(33.3) & $2(18.8)$ & $9(81.8)$ \\
\hline Pseudomonas spp & $2(13.3)$ & 13(86.6) & $3(18.7)$ & 13(81.2) & $8(47)$ & $9(52.9)$ & $1(20)$ & $4(80)$ & 0 & $8(100)$ \\
\hline Escherichia coli & $2(14.2)$ & $12(85.7)$ & $2(14.2)$ & $12(85.7)$ & 13(86.6) & $2(13.3)$ & $1(100)$ & 0 & $2(33.3)$ & $4(66.6)$ \\
\hline Staphylococcus aureus & 0 & $4(100)$ & $1(25)$ & $3(75)$ & $3(50)$ & $3(50)$ & 0 & $2(100)$ & $1(25)$ & $3(75)$ \\
\hline Streptococcus & 0 & $3(100)$ & 0 & $3(100)$ & $1(25)$ & $3(75)$ & $1(50)$ & $1(50)$ & 0 & $2(100)$ \\
\hline Salmonella spp & 0 & $1(100)$ & 0 & $1(100)$ & $1(100)$ & 0 & - & - & - & - \\
\hline Morganella morganii & 0 & $1(100)$ & 0 & $1(100)$ & 0 & $1(100)$ & 0 & $1(100)$ & 0 & $1(100)$ \\
\hline Total & $15(16.6)$ & $75(83.3)$ & 18(19.7) & $73(80.2)$ & $54(54.5)$ & $45(45.4)$ & $7(36.8)$ & $12(63.1)$ & 17(32.6) & $35(67.3)$ \\
\hline
\end{tabular}

Note: S: antibiotic sensitive, R: antibiotic resistant.

\section{Discussion:}

This study provides an analysis of epidemiology and microbiology of infections in the ICU patients of a private medical college hospital in Dhaka. Consistent with other studies, ${ }^{6}$ aspiration pneumonia (49\%), pneumonia (10\%) and UTI (20\%) respectively were the leading form of infection in the subjects of our study. The types of organisms that have emerged as most problematic for patients within the ICU include Acinetobacter spp, Klebsiella spp, Pseudomonas spp, Escherichia coli. ${ }^{6}$ In our study, the most frequent microorganisms derived from samples were Acinetobacter spp (29\%), Klebsiella spp (26\%) and Pseudomonas spp (18\%), Escherichia coli (15\%) respectively which consistent with other study. In recent years Acinetobacter spp. have emerged as important pathogens of ICUs, most of them being resistant to ampicillin, carbenicillin, cefotaxime, chloramphenicol, and gentamicin. ${ }^{7,8}$ In our study, Acinetobacter spp was the number one cause of pneumonia based on samples gathered from the tracheal aspirate and shows resistance to ceftriaxone (85.1\%), ceftazidime (88.8\%), amikacin $(86.2 \%)$, gentamicin (84.4\%) and fluoroquinolones (86.2-89.2\%) respectively. This is consistent with the results of a similar study conducted in India. ${ }^{3,9}$

Another type of commonly seen antimicrobialresistant pathogen among ICU patients is Klebsiella spp, which is producing extended-spectrum betalactamases (ESBLs). ${ }^{3,6}$ Our isolates of Klebsiella spp showed high resistance to broad-spectrum cephalosporins and gentamicin (82.6-84.6\% and $66.6 \%$, respectively). But they were very much sensitive to meropenem (84.6\%).
Gram-negative bacilli are frequently associated with nosocomial infections in ICUs. Data from a multicenter Intensive Care Unit Surveillance Study (ISS) in the United States demonstrated that Pseudomonas spp are frequently isolated from ICU samples and they are especially resistance to fluoroquinolones. ${ }^{10}$ The isolates of Pseudomonas spp in this study were $18 \%$, mostly from tracheal aspirate and urine. In this study, Pseudomonas spp were resistant against Ceftriaxone (70.5\%), Ceftazidime (66.6\%), Amikacin (68.7\%), Gentamicin (58.8\%), Meropenem (52.9\%) and Quinolones (81.2-86.6\%) respectively, which is closely consistent with other study. ${ }^{6}$

In our investigation, E. coli was the most frequent pathogen obtained from patients with urinary tract infection. This is similar to previous study. ${ }^{11}$ In that study, Escherichia coli species were fully susceptible to meropenem, but resistant to ceftriaxone. ${ }^{11}$ In our study, similarly Escherichia coli were mostly sensitive to Meropenem (86.6\%), Amikacin (73.3\%) and resistant to ceftriaxone $(80 \%)$, ceftazidime $(64.2 \%)$ respectively.

Multiresistant klebsiellae, Pseudomonas and Acinetobacter species have given new dimensions to the problem of hospital-associated infections. The panic situation is infection with Acinetobacter spp, where no single antibiotic has shown effective sensitivity.

\section{Conclusion:}

Antibiotics and similar drugs, together called antimicrobial agents, have been used for many years, since first world war to treat patients who had 
infectious diseases. Since the 1940s, these drugs have greatly reduced illness and death from infectious diseases. ${ }^{12}$ Antibiotic use has been beneficial when prescribed and taken correct way. Their value in patient care is enormous. Infections caused by resistant microorganisms often fail to respond to conventional treatment, resulting in prolonged illness, prolong hospital stay and greater risk of death. ${ }^{12}$

A number of factors contribute to the emergence of antimicrobial resistance in ICUs including the severity of patient illness, predisposition to nosocomial infections, cross-transmission of pathogens characteristic of critical care areas within the hospital, compromised membrane and skin barriers following the use of invasive devices, extended length of hospital stay, and the widespread use of prophylactic and therapeutic anti-infective agents. Antimicrobial resistance among intensive care unit (ICU) pathogens is gradually increasing, but it varies from country to country, probably due to individual antimicrobial use patterns. We need institute based surveillance mechanisms in the health sectors to generate reliable and actionable epidemiological information including baseline data and trends on antimicrobial resistance, utilization of antimicrobial agents and impact on the economy and health through designated national and regional reference centers.

Conflict of interest: We have no conflict of interest.

\section{References:}

1. Faiz MA, Basher A. Antimicrobial resistance: Bangladesh experience. Regional Health Forum 2011; 15(1): 1-8.

2. Silveira F, Fujitani F, Paterson DL. Antibioticresistant infections in the critically ill adult. Clin Lab Med 2004; 24: 329-341.

3. Jamshidi M, Javadpour S, Eftekhari TE, Moradi N, Jomehpour F. Antimicrobial resistance pattern among intensive care unit patients. African Journal of Microbiology Research 2009; 3(10):590-594.

4. Ewans TM, Ortiz CR, LaForce FM. Prevention and control of nosocomial infection in the intensive care unit. In: RS Irwin, FB Cerra, JM Rippe (eds.), Intensive care medicine, Lippincot-Raven, New York pp. (1999): 1074-1080.

5. Wenzel RP, Thompson RL, Landry SM, Russell BS, Miller PJ, Ponce DL. Hospital-acquired infections in intensive care unit patients: an overview with emphasis on epidemics. Infect. Control 1983; 4: 371-375.

6. Bayram A, Balci I. Patterns of antimicrobial resistance in a surgical intensive care unit of a university hospital in Turkey. BMC Infectious Diseases 2006; 6:155 [doi:10.1186/1471-2334-6-155].

7. Lortholary O, Fagon JY, Hoi AB, Slama MA, Pierre J, Giral P, Rosenzweig R, Gutmann L, Safar M, Acar $\mathrm{J}$ : Nosocomial acquisition of multi-resistant Acinetobacter baumannii: Risk factors and prognosis. Clin Infect Dis 1995; 20:790-796.

8. Seifert H, Baginski R, Schulze A, Pulverer G: Antimicrobial susceptibility of Acinetobacter species. Antimicrob Agents Chemother 1993; 37:750-753.

9. Kumari HB, Nagarathna S, Chandramuki A. Antimicrobial resistance pattern among aerobic gramnegative bacilli of lower respiratory tract specimens of intensive care unit patients in a neurocentre. Indian J. Chest. Dis. Allied. Sci. 2007; 49: 19-22.

10. Friedland I, Gallagher G, King T, Woods GL. Antimicrobial susceptibility patterns in Pseudomonas aeruginosa: data from a multicenter Intensive Care Unit Surveillance Study (ISS) in the United States. $J$ Chemother 2004; 16:437-441.

11. Kiffer C, Hsiung A, Oplustil C, Sampaio J, Sakagami E, Turner P. Antimicrobial susceptibility of Gramnegative bacteria in Brazilian hospitals: the MYSTIC Program Brazil 2003. Braz. J. Infect. Dis. 2005; 9: 216-224.

12. Islam QT. Antimicrobial Resistance: A Man Made Crisis. J Bangladesh Coll Phys Surg 2011; 29: 120125. 